\title{
Comparative study on predicting Young's modulus of graphene sheets using nano-scale continuum mechanics approach
}

\author{
Roham Rafiee*, Amirali Eskandariyun
}

\begin{abstract}
In this research, nano-scale continuum modeling is employed to predict Young's modulus of graphene sheet. The lattice nano-structure of a graphene sheet is replaced with a discrete space-frame structure simulating carbon-carbon bonds with either beam or spring elements. A comparative study is carried out to check the influence of employed elements on estimated Young's moduli of graphene sheets in both horizontal and vertical directions. A detailed analysis is also conducted to investigate the influence of graphene sheet sizes on its Young's modulus and corresponding aspect ratios that unwelcomed end effects disappear on the results are extracted. At the final stage, defected graphene sheets suffering from vacancy defects are investigated through a stochastic analysis taking into account both number of defects and their locations as random parameters. The reduction level in the Young's moduli of defected graphene sheets compared with non-defected ones is analyzed and reported.
\end{abstract}

Keywords: Graphene; Continuum modeling; Young's modulus; Stochastic analysis; Vacancy defects

\section{Introduction}

A new generation of polymeric composites has blossomed over the last decades owning to the discovery of nano-materials as a new generation of reinforcing agents. Among them, a considerable attention have been devoted to carbon nanotubes (CNT) and graphene sheets due to their extraordinary and unique properties from different mechanical, electrical and thermal aspects. Although CNTs and graphene sheets present almost the same class of mechanical properties, graphene is preferred to CNT when certain thermal and electrical properties are focused [1].

A fundamental step prior to understanding mechanical behavior of graphene reinforced polymers is characterizing mechanical properties of isolated graphene. Tremendous limitations exposed to the context of nano-scale experimental measurements have stimulated researchers to pursue different theoretical 
modeling techniques. Generally, modeling techniques at nanoscale are classified into three main categories of atomistic modeling, continuum modeling and nano-scale continuum modeling [2]. Atomistic modeling methods are suffering from intensive computational efforts and complicated formulations. Moreover, they cannot be applied to the systems with large numbers of atoms. Continuum models are constructed on the basis of Elasticity theories by replacing a lattice structure of CNT/graphene with a simple continuum medium. The validity of continuum modelings are required to be studied carefully where a real discrete structure of CNT/graphene is neglected. Nano-scale continuum models are identified as those modeling techniques where continuum assumptions are applied to the molecular bonds instead of the whole nano-structure [2]. In these techniques, each $\mathrm{C}-\mathrm{C}$ bond is replaced with a continuum element while the discrete nature of the nano-structure is not violated. The replacement of $\mathrm{C}-\mathrm{C}$ bonds with equivalent elements appearing in the variety forms of beam, rod, truss or spring is accomplished by establishing a linkage between molecular configurations and solid mechanics. Different researchers have used nano-scale continuum mechanics to analyze the mechanical behavior of CNTs using either linear or nonlinear elements [3-17]. The majority of nano-scale modeling techniques have been carried out numerically, while very rare studies have been performed analytically [4-6,13]

Limited studies have been concentrated specifically on graphene sheets in literature. Meo and Rossi have modeled graphene sheets employing translation and torsional spring element for simulating bond stretching and bond angle variations. They have employed non-linear interatmoic potenrials in their study [9]. Shokrieh and Rafiee [13] have developed a closed form solution for predicting Young's moduli of a graphene sheet in two orthogonal directions substituting the lattice structure of a graphene sheet with a honeycomb-like discrete structure. In some studies, the Young's modulus of a graphene sheet is evaluated employing atomistic modeling techniques $[18,19]$. Some other investigators have originally predicted the Young's modulus of CNTs versus CNT radius and then estimated the Young's modulus of a graphene sheet relying on this fact that a graphene sheet can be virtually viewed as a CNT with infinite radius [4, 6]. Zhang et el. [20] have predicted the mechanical properties of a bilayer graphene sheet coupled by $\mathrm{sp}^{3}$ bonding using molecular dynamics (MD) simulation. Investigating the influence of Stone-Wales defects on the tensile properties of a graphene sheet, Ren an Cao [21] have reported that this type of defect does not have any influence on the Young's modulus and Poisson's ratio using density functional theory. Elastic mechanical properties of graphene sheets have been computed by Georgantzinos and his co-workers using spring elements and incorporating either linear or nonlinear interatomic potential [22, 23]. Recently, Javvaji et al. [24] have extensively investigated the effect of domain size, lattice orientation and crack length on the mechanical properties of graphene employing MD simulations.

The main objective of this article is to perform a comparative study on different nano-scale continuum mechanics approaches for extracting Young's modulus of graphene nanostructure. The influence of linear and non-linear models on the results will be also investigated. Moreover, both defected and non-defected 
graphene sheets are simulated and analyzed to investigate the degree to which structural defect will influence the Young's modulus on the basis of employed modeling technique.

\section{Morphology of graphene sheets}

Graphene layers is considered as the building units of Graphite which are stacked together with interlayer spacing of $0.34 \mathrm{~nm}$ [2]. Graphene can be imagined as a single layer of covalently bonded carbon atoms arranged in the periodic hexagon rings resembling honeycomb lattice configuration (See Fig. 1). The length of carbon-carbon (C-C) bond in graphene layer is nearly $0.142 \mathrm{~nm}$.
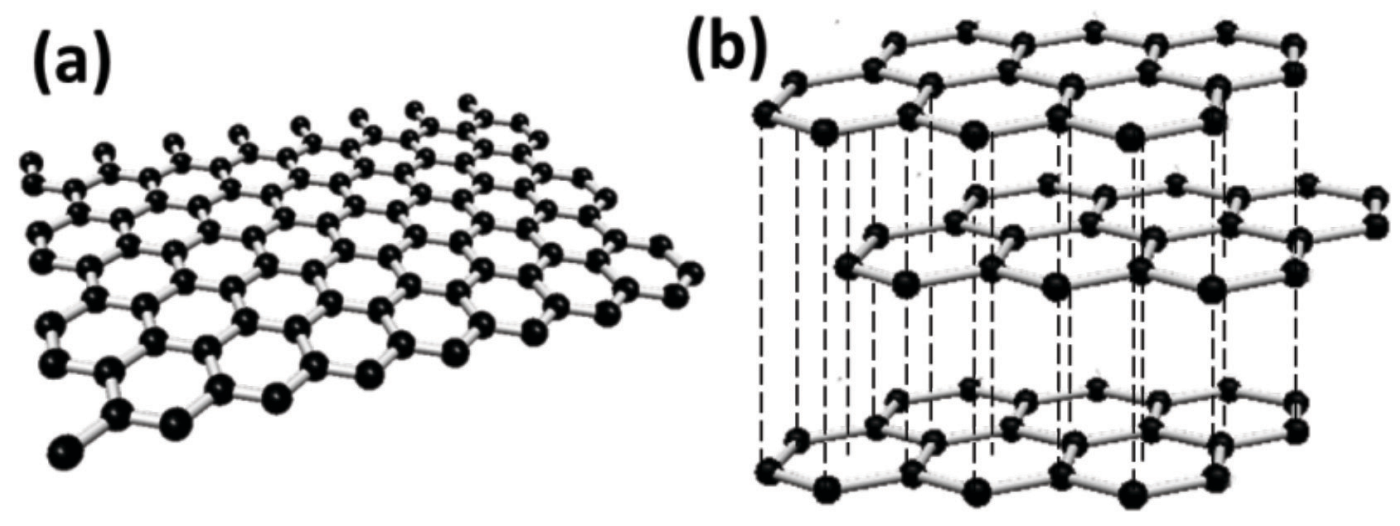

Fig. 1: (a) Lattice nano-structure of a graphene sheet, (b) Graphite consisting of stacked graphene sheets

\section{Molecular interactions}

In the context of molecular studies, total inter-atomic potential energy of a molecular system is regulated by the contribution of individual bonding and nonbonding interactions as below [25]:

$$
E_{t o t}=U_{r}+U_{\theta}+U_{\varphi}+U_{\omega}+U_{V d W}+U_{e s}
$$

where $U_{r}, U_{\theta}, U_{\varphi}$, and $U_{\omega}$ are representative of bond stretching, bond angle variation, dihedral angle torsion and out-of-plane torsion, respectively. $U_{V d W}$ and $U_{e s}$ are associated with non-bonded van der Waals $(\mathrm{vdW})$ and electrostatic interactions. Non-bonded interactions are negligible in comparison with bonded ones. The merged form of dihedral angle torsion and out-of-plane angle torsion is expressed as below [25]:

$$
U_{\tau}=U_{\varphi}+U_{\omega}
$$

Thus, total inter-atomic potential energy of a nano-structure is reduced to contain bond stretching $\left(U_{r}\right)$, bond angle variation $\left(U_{\theta}\right)$ and torsional $\left(U_{\tau}\right)$ terms. Among bonded interactions, the dominant parts of inter-atomic potential are bond stretching and bond angle variations due to their significant contribution in 
comparison with other interactions especially when the graphene is subjected to small in-plane deflections.

Linear representation of total interatomic potential energy of a molecular system can be approximated using harmonic functions as below:

$$
E_{\text {tot }}^{L} \cong U_{\rho}+U_{\theta}+U_{\tau}=\frac{1}{2} k_{r}(\Delta r)^{2}+\frac{1}{2} k_{\theta}(\Delta \theta)^{2}+\frac{1}{2} k_{\varphi}(\Delta \varphi)^{2}
$$

where, reflected force constant in above equation are introduced as below using AMBER force field [26]:

$$
\begin{aligned}
& k_{r}=938\left[\frac{\mathrm{kcal}}{\mathrm{mole}} \times \frac{1}{\mathrm{~nm}^{2}}\right]=6.52 \times 10^{-7}\left[\frac{\mathrm{N}}{\mathrm{nm}}\right] \\
& k_{\theta}=126\left[\frac{\mathrm{kcal}}{\mathrm{mole}^{2}} \times \frac{1}{\mathrm{rad}^{2}}\right]=8.76 \times 10^{-10}\left[\frac{\mathrm{N} . \mathrm{nm}}{\mathrm{rad}^{2}}\right] \\
& k_{\varphi}=40\left[\frac{\mathrm{kcal}}{\mathrm{mole}} \times \frac{1}{\mathrm{rad}^{2}}\right]=2.78 \times 10^{-10}\left[\frac{\mathrm{N} . \mathrm{nm}}{\mathrm{rad}^{2}}\right]
\end{aligned}
$$

Non-linear representation of total interatomic potential energy is estimated using modified Morse potentials as below [27]:

$$
E_{\text {tot }}^{N L} \cong U_{\rho}+U_{\theta}=D_{e}\left\{\left[1-e^{-\beta\left(r-r_{0}\right)}\right]^{2}-1\right\}+\frac{1}{2} k_{\theta}\left(\theta-\theta_{0}\right)^{2}\left[1+k_{\text {sextic }}\left(\theta-\theta_{0}\right)^{4}\right]
$$

where :

$$
\begin{aligned}
& D_{e}=6.03105 e^{-19}[N . m], \beta=2.625 e^{10}\left[m^{-1}\right], r_{0}=0.142[\mathrm{~nm}] . \\
& k_{\theta}=0.9 e^{-18}\left[N \cdot \frac{\mathrm{m}}{\mathrm{rad}^{2}}\right], k_{\text {sextic }}=0.754\left[\mathrm{rad}^{-4}, \mathrm{\theta}_{0}=2.094[\mathrm{rad}]\right.
\end{aligned}
$$

In the next section, $E_{\text {tot }}^{L}$ and $E_{\text {tot }}^{N L}$ are used separately to construct linear and non-linear Finite Element (FE) models of a graphene sheet, respectively.

\section{Finite element modeling}

In the field of nano-scale continuum modelings, there are two broadly used strategies in modeling CNT or graphene nano-structure recognized as beam-based or spring-based models. Following the fundaments of nano-scale continuum modeling techniques, the later uses spring elements to capture mechanical behavior of $\mathrm{C}-\mathrm{C}$ bonds while the former exploits beam elements for constructing FE model. The fundaments of these modeling techniques are outlined in this section which will be used for the purpose of comparative study. It is worth mentioning that in both categories, linear and non-linear elements can be used.

A computer code is written in APDL of ANSYS to construct geometrical model of a graphene sheet. In this model, structural nodes are placed at the exact location of carbon atoms in graphene sheet. 


\subsection{Beam-based FE modeling}

In beam-based FE modeling which was originally developed by Li and Chou [28] for modeling CNT, each C-C bond is replaced with a linear beam element. The mechanical properties and sectional parameters of this beam element are obtained using an analogy between interatomic potential energies in molecular space and strain energies in structural mechanics. Correlating bond stretching with the axial deformation of beam, bond angle variation with beam bending and also bond torsion with beam torsion, the below formulations are derived [28]:

$$
\frac{E A}{L}=k_{r} \quad, \quad \frac{E I}{L}=k_{\theta} \quad, \quad \frac{G J}{L}=k_{\varphi}
$$

The 3D linear beam element in ANSYS is used for constructing linear beam-based FE model. Young's modulus and diameter of the beam element are obtained using Eq. (8). Isotropic behavior is assumed for the beam element and thus the Poisson's ratio is obtained.

The coordinates of each carbon atom on a graphene sheet are fed into ANSYS as the nodal coordinate of the model and appropriate nodes are connected using abovementioned beam element. Tserpes et al. [29] have developed a stepwise procedure of progressive modeling for modeling CNT using non-linear beam elements. In their model, the initial stiffness of the beam elements are calculated from the initial slope of the force-strain curve explained by modified Morse potential (the first term reflected in Eq. 5). Then, the stiffness of each C-C element is updated at each load step on the basis of the induced axial strain in each element. Due to the very time consuming procedure of this modeling technique, in this research, just linear beam-based FE model is constructed.

\subsection{Spring-based FE modeling}

Beam-based FE modeling is suffering from two shortcomings: (i) C-C bonds remain straight in deformed shapes of CNT or graphene while beam element will undergo bending, (ii) it is necessary to assign some geometrical characteristics like diameter, area and moment of inertia to the beam elements while the real nature of $\mathrm{C}-\mathrm{C}$ bonds is independent from these non-physical parameters. Therefore, Spring-based FE modeling is introduced to overcome aforementioned shortcomings.

Two approaches have been developed for construction of spring-based FE models for CNT. Meo and Rossi have simulated CNT using translational springs to capture bond stretching while torsional springs are used for bond angle variations [9]. Giannopolus et al. [10] have simulated CNT using just translational spring elements. They have used two different translational springs for capturing both bond stretching and bond angle variations.

The developed strategy by Giannopolus et al. [10] is used here to construct spring-based FE model of a graphene and it is briefly explained. In this method, each $\mathrm{C}-\mathrm{C}$ bond is replaced with a translational spring elements representing bond stretching. These 6 spring elements are known as "A" group. Another 6 spring elements categorized as group "B" accounts for bond angle variation. These spring elements 
connect two opposite carbon atoms at the end of two adjacent C-C bonds. Any variation in the length of B-spring elements implies on the angle variations of between two adjacent $\mathrm{C}-\mathrm{C}$ bonds. Both A- and Bspring elements are shown in Fig. 3 in comparison with previously explained beam-based model.

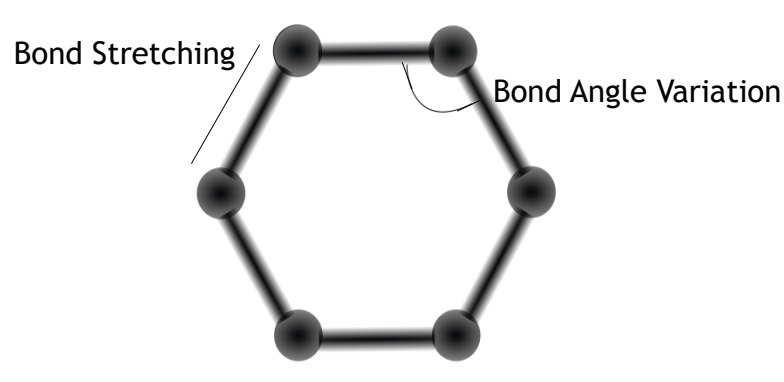

(a)

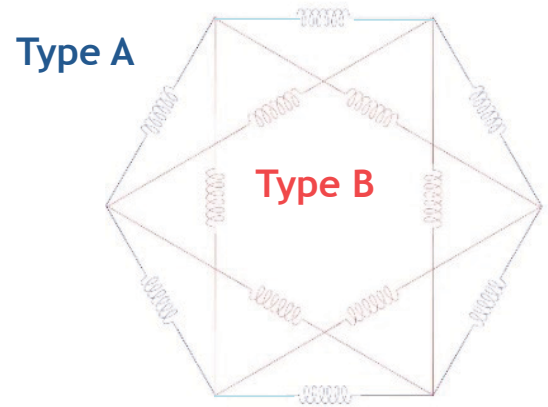

(b)

Fig. 3: One hexagon unit of a graphene sheet simulated by (a) beam elements and (b) spring elements

Recalling from Fig. 3, for small deformation, variations in the length of B-spring elements can be related to the bond angle variation using below equation [10]:

$$
\Delta \theta=\frac{2(\Delta R)}{r_{0}}
$$

where $r_{0}$ is the initial length of $\mathrm{C}-\mathrm{C}$ bond and $\Delta R$ is the change in the length of B-spring element. For the linear model, spring constants are calculated using Eqs. (4) and for the non-linear model, Eqs. (6) and (7) are used.

Generally, three FE models, i.e. linear beam-based, linear and non-linear spring-based models are built for the purpose of comparative study. Both beam- and spring-based FE models of the graphene sheet are presented in Fig. 4.

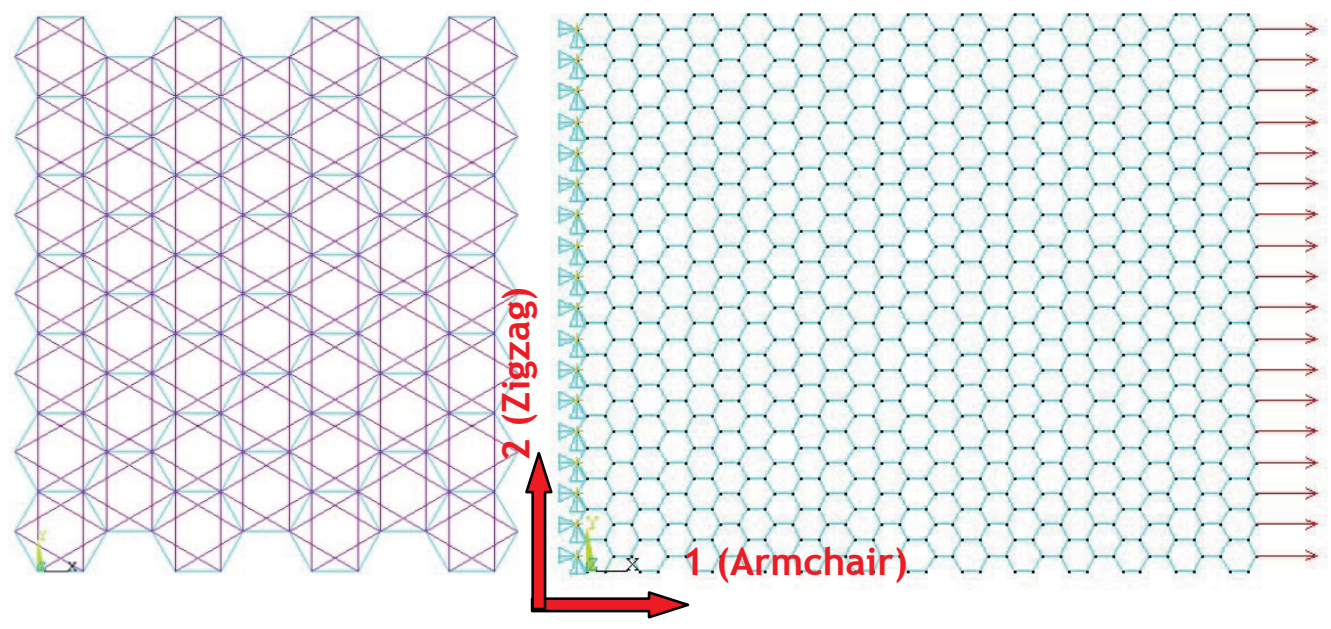


Fig. 4: Spring-based (left) and beam-based (right) FE models of a graphene sheet

\section{Evaluating the Young's modulus of graphene}

In both linear and non-linear models, one end is fixed while axial deformation is applied to other end (Fig.

4). The FE models are analyzed in two orthogonal directions to obtain both longitudinal and transverse moduli for graphene sheets. For the case of non-linear model, full Newton-Raphson method is used for non-linear analysis and incremental solution is conducted by selecting a very small load increment. Since, the non-linear model are able to capture $\mathrm{C}-\mathrm{C}$ bond breakage, small deformation is applied preventing any bond breakage during the analysis.

The Young's moduli of analyzed graphene sheets in both (1) and (2) directions are calculated using below formulations:

$$
\begin{aligned}
& E_{A}=\frac{F_{1} L}{W \cdot t \cdot \Delta L} \\
& E_{Z}=\frac{F_{2} W}{L . t . \Delta W}
\end{aligned}
$$

where F's are resultant force read from the output of FE analysis. L and W is the length of graphene sheet along (1) and (2) direction, respectively. It should be pointed out that directions (1) and (2) are referred to as Armchair and Zigzag graphenes.

Firstly, the aspect ratio of the graphene is chosen as unity (i.e. $\mathrm{L}=\mathrm{W}$ ) and dimensions are varied to find a region where numerical errors caused by the effects of boundary conditions on the overall structural behavior diminish. The observed trends for both linear models are presented in Fig. 5 .
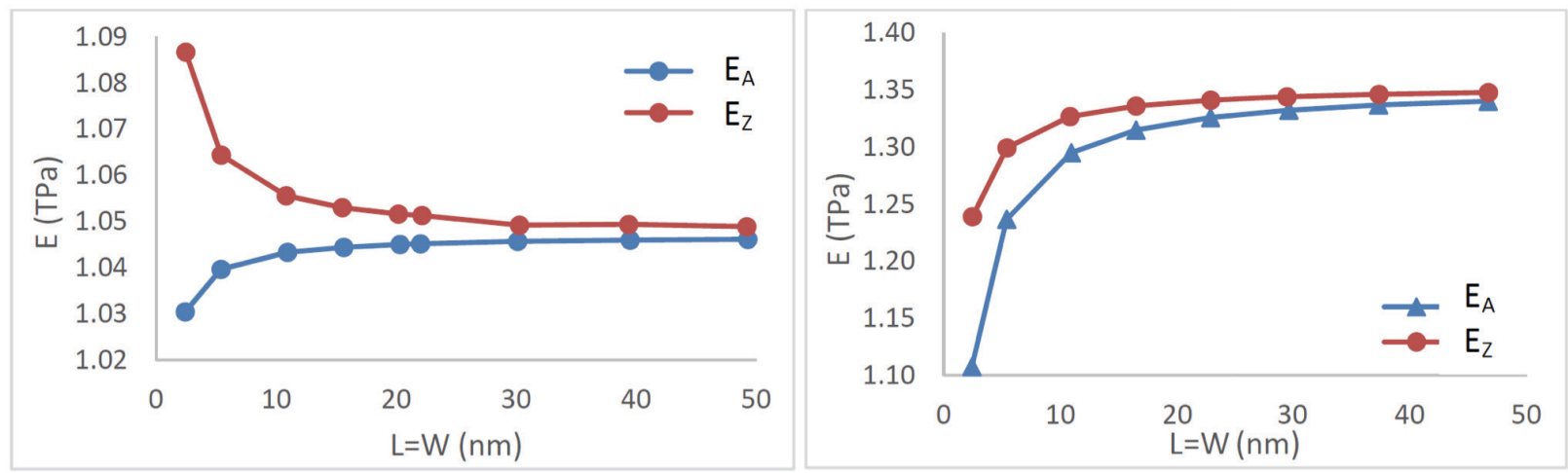

Fig. 5: Young's modulus of graphene sheet vs. graphene size linear beam model (left), linear spring model (right)

The observed different trends of convergence in Fig. 5 for beam and spring model is originated from the different behavior of the beam and spring elements. It is evident from the results that when the graphene length exceeds $30 \mathrm{~nm}$, the unwelcomed numerical errors arisen from end effects disappears. 
It has been reported in literature that Young's modulus and Poisson's ratio of a graphene nanoribbon depend strongly on its size and chirality using MD simulation [30]. Although the dependency of the Young's modulus of a graphene sheet to its size can be considered as its inherent behavior at nanoscale, the authors believe that the reported dependency of graphene Young's modulus to its size in the current study is arisen from the unwelcomed end effect as a consequence of Saint-Venant's principle using continuum modeling [31]. It was also reported in literature that in continuum modeling of CNT, the dependency of CNT Young's modulus to its aspect ratio (L/D) vanishes when the aspect ratio is larger than 10 [32-34].

A comparison between obtained Young's modulus of analyzed graphene sheets for all three models are presented in Table 1. It should be pointed out that inserted Young's moduli for the non-linear spring model are the initial moduli obtained at very small strains. A comparison between obtained results in present study with reported results in literature is also summarized in Table 1 accompanied with employed or developed modeling techniques. Moreover, a very close values for Young's modulus in both directions imply on isotropic behavior of all graphene models. In other words, replacing $\mathrm{C}-\mathrm{C}$ bonds on a graphene sheet will lead us to isotropic behavior when the length of graphene is more than $30 \mathrm{~nm}$. In contrast, when the length is below $30 \mathrm{~nm}$, anisotropic behavior is evident for all linear beam, linear spring and non-linear spring models. 
Table 1: Comparison of Young's modulus for graphene sheets

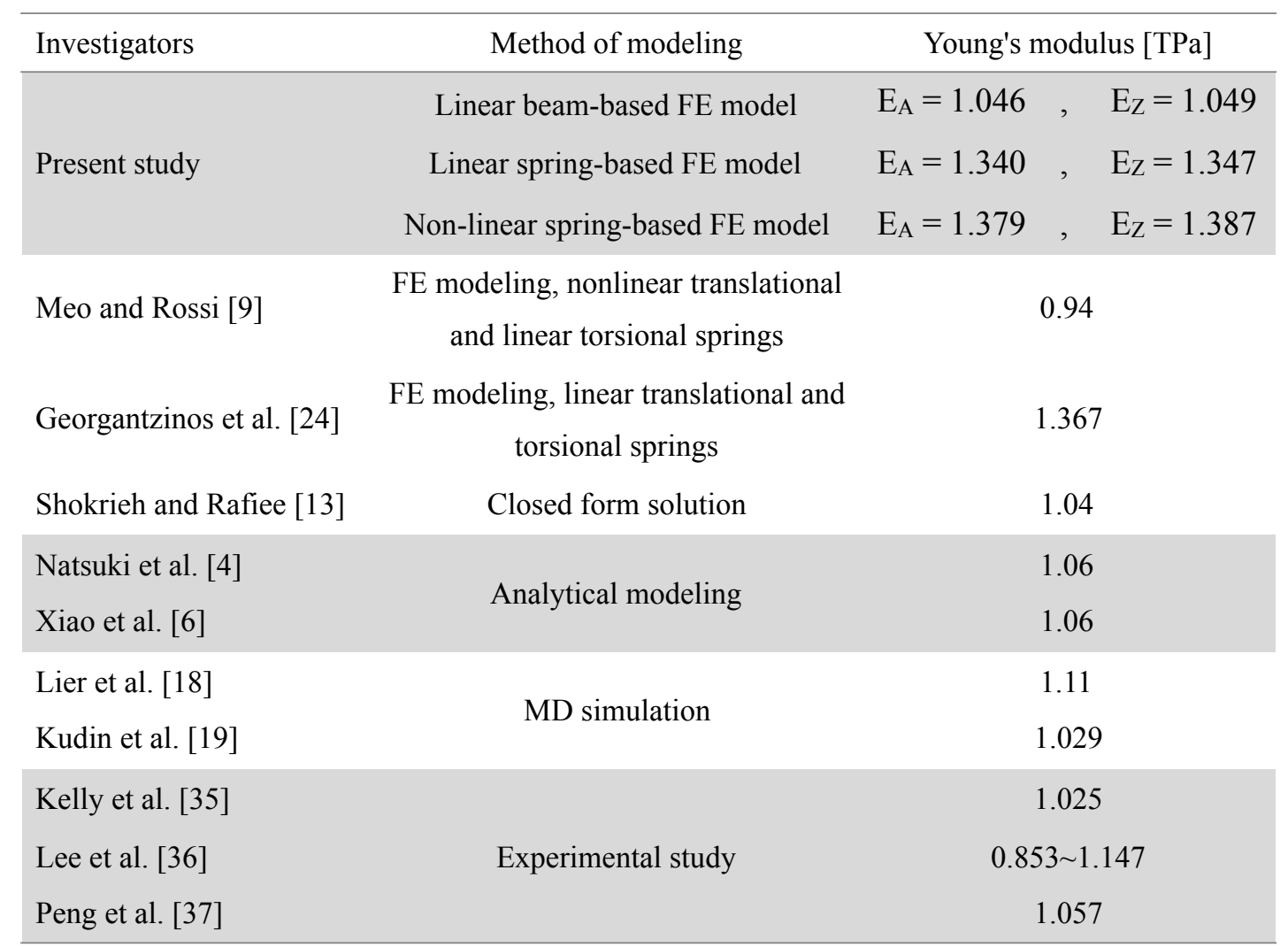

As it can be understood from reported results in Table 1, the Young's modulus of spring-based graphene is $28 \%$ higher than that of beam-based model. This can be justified by this fact that those spring elements responsible to capture bond angle variations (Type B) also contributes in axial deformation of the graphene and thus at the same level of strain, higher values of resultant forces are reported. Furthermore, nonlinear spring models also predict slightly higher values of Young's moduli in comparison with linear spring model. This is originated from the different employed interatomic potential. The same trend was also reported for the case of CNT Young's modulus employing linear and non-linear interatomic potentials [15].

In the next stage, the influence of graphene sheet size on the Young's modui of graphene sheet is studied. Due to the small difference between reported results for non-linear and linear spring-based models (about $3 \%$ ), both linear beam and spring models are hereinafter concentrated. For this purpose, the width or length is kept constant while another dimension is varied to investigate the effect of various aspect ratios (length/width) of the graphene sheets on the estimated Young's moduli. The obtained results are depicted in Fig. 6. It can be seen that for both models, plateau behaviors are observed when the aspect ratio is between 0.4 and 2.5 resembling those sizes where one dimension is two-and-a-half times greater than the other. 


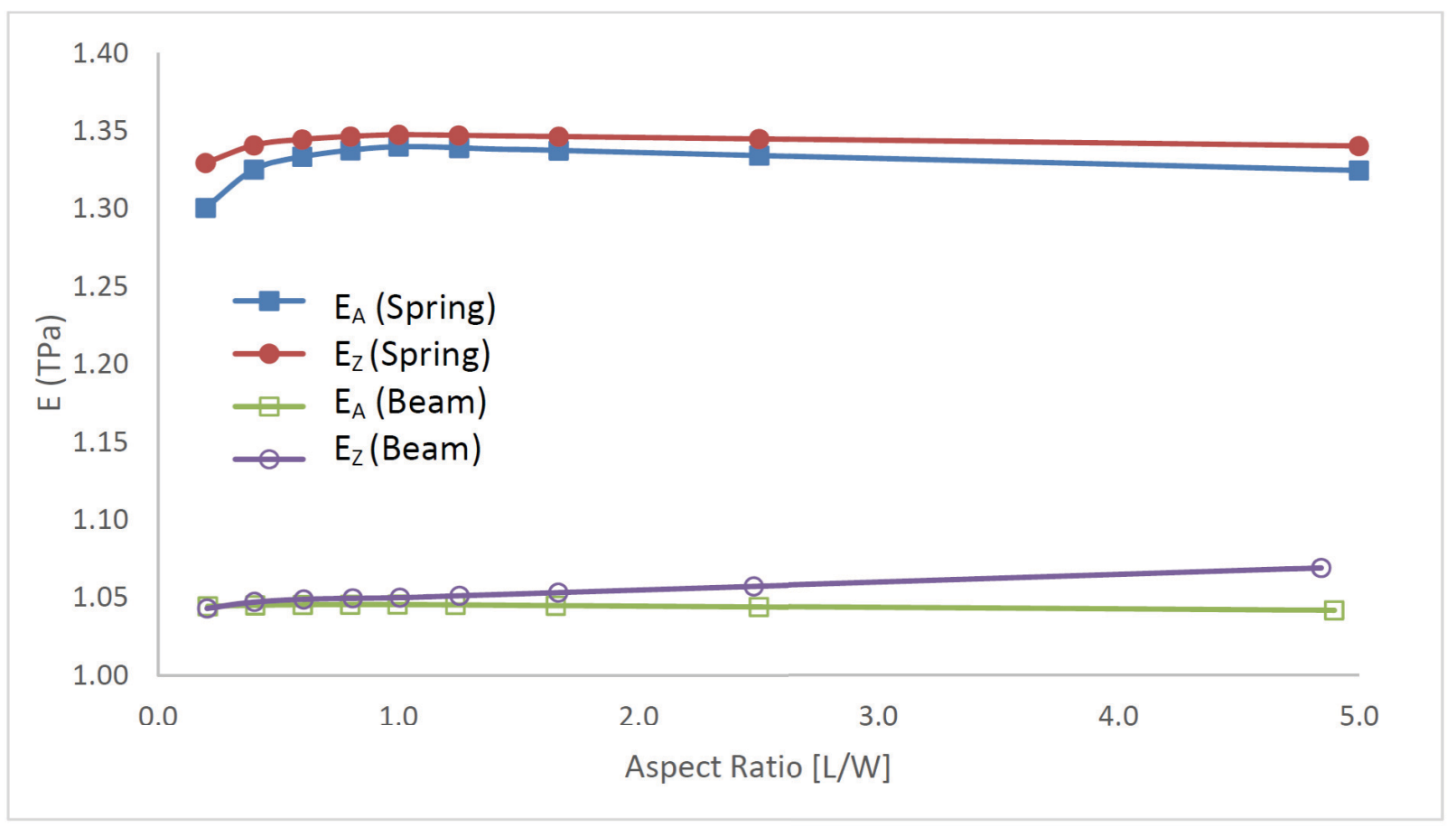

Fig. 6: The influence of aspect ratio on the Young's moduli of graphene sheet

\section{Modeling defected graphene sheet}

The influence of vacancy defects on the Young's modulus of graphene sheet and the degree to which the modulus is reduced is investigated in this section. For this purpose, stochastic modeling is employed using Monte-Carlo method. A macro is written in APDL of ANSYS to randomly generate vacancy defects in the nano-structure of graphene by removing $\mathrm{C}-\mathrm{C}$ bonds. The number of defects is also considered as other random variable. Thus, both number of defects and its locations are treated as random parameters. The flowchart of employed stochastic modeling for extracting Young's modulus of defected graphene sheets is presented in Fig. 7. 


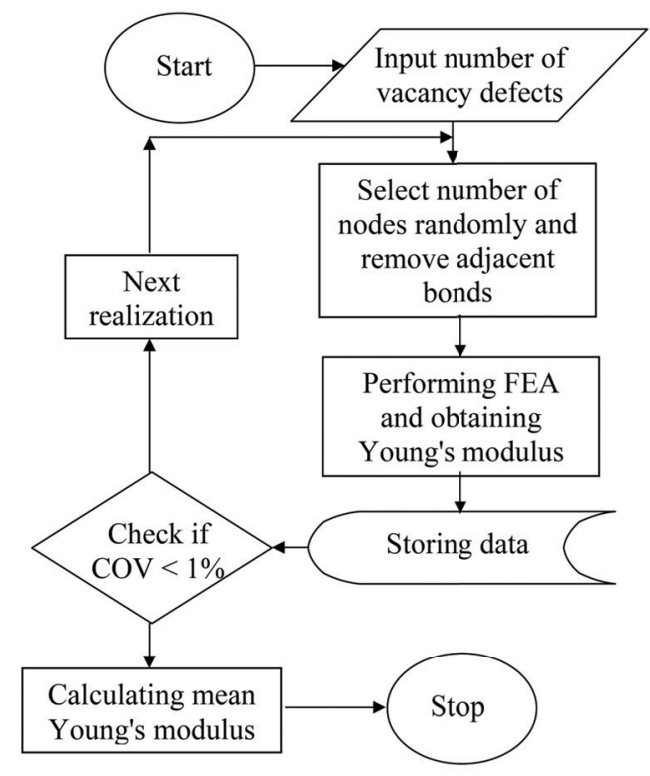

Fig. 7: Flowchart of stochastic modeling of defected graphene sheets

Generated FE models of defected graphene is subjected to the same boundary conditions explained in section (4). For each specific numbers of defects, sufficient numbers of realizations are generated because of random nature of FE modeling procedure. Obtaining the coefficient of variations (COV) for calculated Young's modulus less than $1 \%$, the generation of random samples is terminated. As a result, the average of Young's modulus is reported. The explained procedure is executed on linear spring and beam models, since it has been already mentioned in the preceding section that linear and non-linear spring models address very close values. Two FE models of graphene sheets with vacancy defects are presented in Fig. 8.

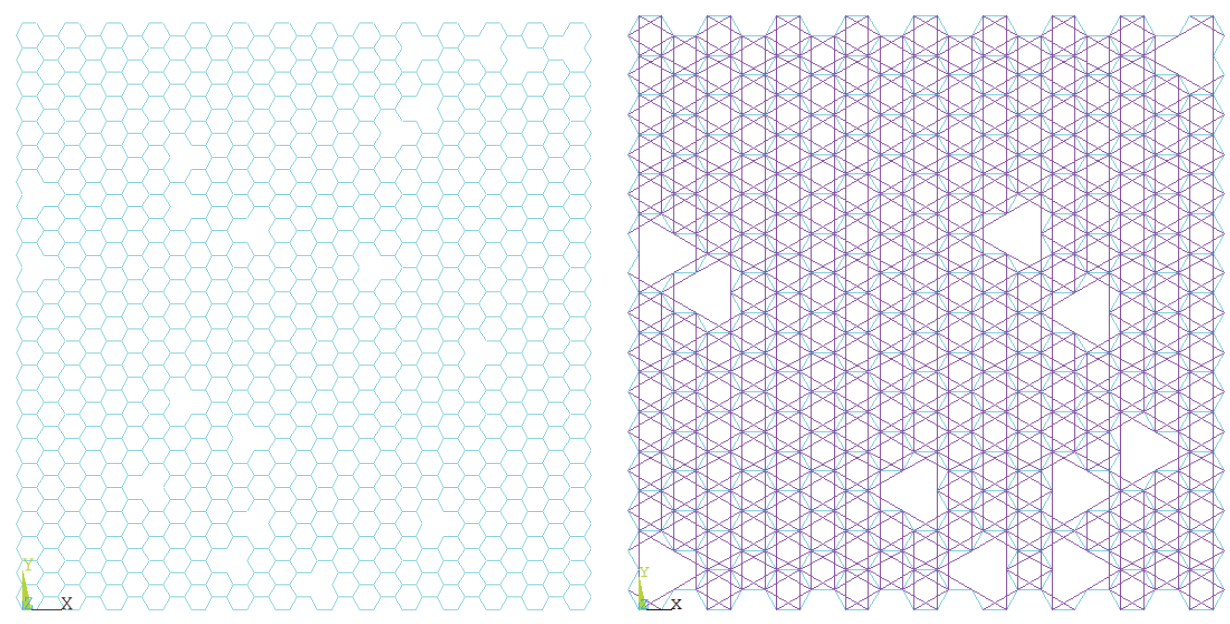

Fig. 8: Defected FE models of graphene sheets 
According to the observed dependency of graphene Young's modulus to its dimensions, in the preceding section, the investigation is performed on the large graphene sheets with unit aspect ratio. A defect density parameter is also defined as the percentage of broken $\mathrm{C}-\mathrm{C}$ bonds to the whole $\mathrm{C}-\mathrm{C}$ bonds in a defected graphene sheets. A monotonically linear decreasing trend for the reduction in graphene Young's modulus versus defect density is observed for both models and also presented in Fig. 9. Very similar reduction trends are observed for both beam and spring models. It can be interpreted from the results that the induced vacancy defects significantly reduce Young's modulus of graphene sheet and it is necessary to take it into account for estimating mechanical properties of graphene-based nanocomposites.

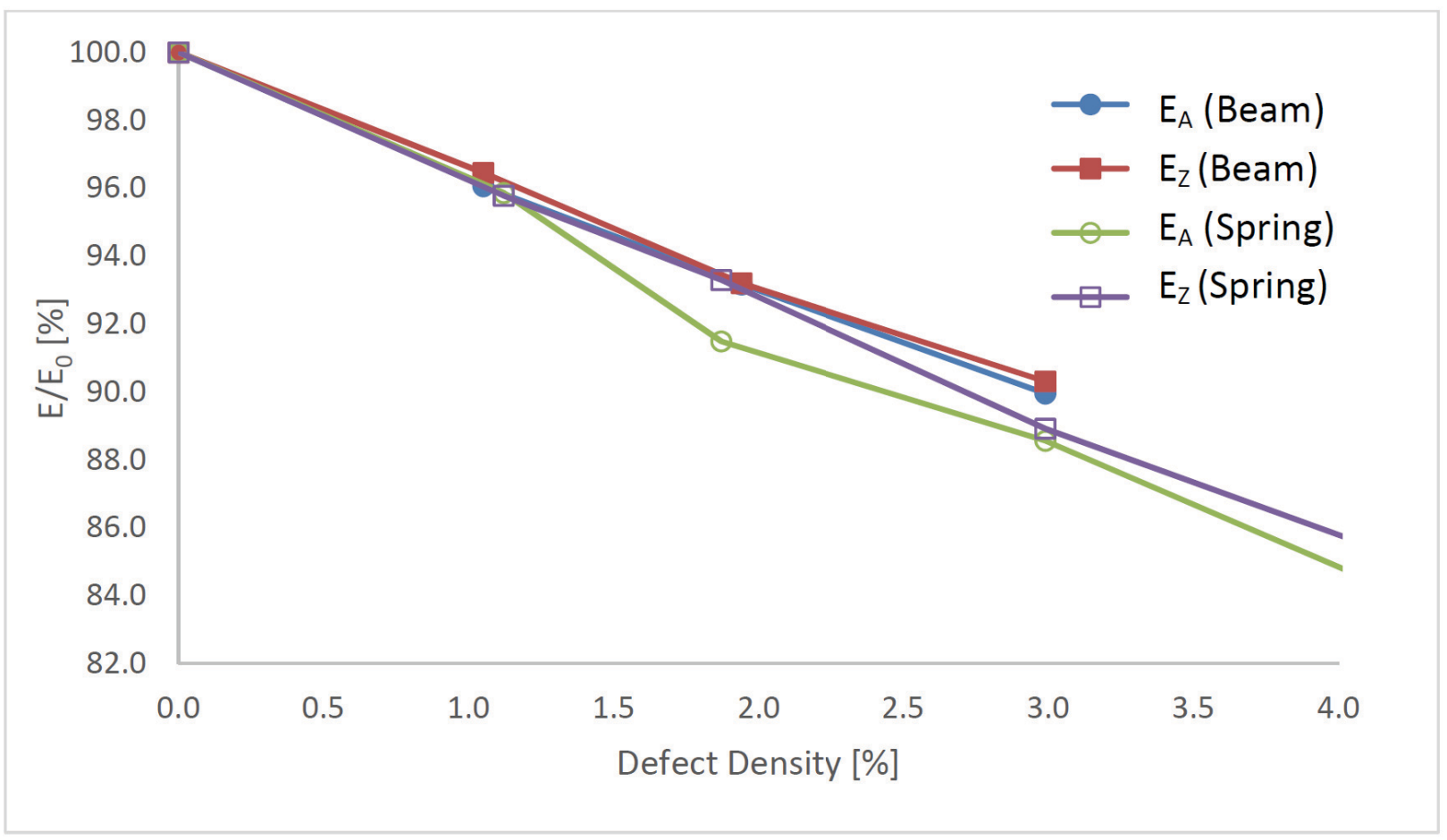

Fig. 9: Decreasing trends of Young's modulus for defected graphene sheets

A comparison between obtained results in this research for defected graphene and other data published in literature using MD simulations is provided in Table 2. It can be seen that in present study, almost linear reduction trend is observed due to the employed linear interatomic potentials; while in some MD simulation non-linear trend is observed because of non-linear potential utilized in atomistic simulation. Different observed values in MD simulations are originated from various interatomic potential used at molecular level. 
Table 2: Comparison of reduction in Young's modulus of defected graphene with the results of MD simulations

\begin{tabular}{lcc}
\hline & Reduction at $1 \%$ defect density & Reduction at 3\% defect density \\
\hline Present Work (Beam-based) & $4 \%$ & $10 \%$ \\
Present Work (Spring-based) & $4 \%$ & $11 \%$ \\
Hao et al. [38] & $3 \%$ & $8.5 \%$ \\
Zhu et al. [39] & $2.5 \%$ & $7.5 \%$ \\
Ito et al. [40] & $5 \%$ & $15 \%$ \\
Anastasi et al. [41] & $2.5 \%$ & $14 \%$ \\
\hline
\end{tabular}

\section{Conclusions}

A comparative study is conducted on predicting Young's modulus of graphene sheets using different elements relying nano-scale continuum mechanics. Three different models are constructed replacing C-C bonds with linear beam, linear and nonlinear spring elements are constructed. FE analysis is conducted to estimate Young's modulus of graphene sheets in both horizontal and vertical directions. The results show that when the unwelcomed effects of numerical errors attributed to the end effect are eliminated by increasing the size of the graphene sheet, isotropic behavior is observed in both beam-based and springbased models. A detailed study is conducted on the influence of graphene size on estimated Young's modulus and it is evident that for small aspect ratios (either $\mathrm{L} / \mathrm{W}$ or $\mathrm{W} / \mathrm{L}$ ), both models address different Young's modulus in horizontal and vertical directions.

Generally, the beam based model addresses lower Young's moduli in comparison with spring-based ones arisen from induced bending in beam elements while spring elements remain straight. due to the difference between linear and non-linear interatomic potentials, the non-linear spring models also report slightly higher Young's moduli compared with linear spring model.

As a rule of thumb, the beam-based models can be easily used when linear analysis is considered; while for non-linear analysis utilizing spring-based models are more convenient. Since, there is not any consistent data for the Young's modulus of graphene sheet either experimentally or theoretically, it can be judged whether beam-based models underestimate the results of spring-based ones overestimate the Young's moduli. The influence of these two elements on estimated Young's moduli have to be taken into account.

Finally, a stochastic analysis is performed to study the degree to which induced vacany defects can reduce the Young's moduli of graphene. Both defect density and position are assumed as random paramters. The results reveal that A linear reduction trend for the Young's modulus versus defect density can be taken into account with a good level of accuracy. 


\section{Acknowledgment}

The authors acknowledge the financial support provided by the Iranian National Science Foundation (INSF) under contract 94003368.

\section{References:}

1.T. Kuilla, S. Bhadra, D. Yao, N. H. Kim, S. Bose, J. H. Lee, Recent advances in graphene based polymer composites, Progress in Polymer Science 2010; 35: 1350-1375.

2.R. Rafiee, R. Maleki Moghad, On the modeling of carbon nanotubes: a critical review, Compos. Part BEng. 2014; 56:435-449.

3.C. Li, T.-W. Chou, Elastic moduli of multi-walled carbon nanotubes and the effect of van der Waals forces. Compos Sci Technol 2003;63:1517-24.

4.T. Natsuki T, K. Tantrakarn, M. Endo, Prediction of elastic properties for single walled carbon nanotubes. Carbon 2004;42:39-45.

5.T. Natsuki, M. Endo, Stress simulation of carbon nanotubes in tension and compression. Carbon 2004;42:2147-51.

6.J.R. Xiao, B.A. Gama BA, Jr J.W. Gillespie, An analytical molecular structural mechanics model for the mechanical properties of carbon nanotubes. Int J Solids Struct 2005;42:3075-92.

7.K.I. Tserpes, P. Papanikos, Finite element modeling of single-walled carbon nanotubes. Composites: Part B 2005;36:468-77.

8.B. Jalalahmadi, R. Naghdabadi, Finite element modeling of single-walled carbon nanotubes with introducing a new wall thickness. J Phys: Conf Ser 2007;61:497-502.

9.M. Meo, M. Rossi, Prediction of Young's modulus of single wall carbon nanotubes by molecularmechanics based finite element modeling. Compos Sci Technol 2006;66:1597-605.

10.G.I. Giannopoulos, P.A. Kakavas N.K. Anifantis, Evaluation of the effective mechanical properties of single-walled carbon nanotubes using a spring based finite element approach. Comput Mater Sci 2008;41(4):561-9.

11.P. Papanikos, D.D. Nikolopoulos, K.I. Tserpes, Equivalent beams for carbon nanotubes. Comput Mater Sci 2008;43:345-52.

12.A.F. Ávila, G.S.R. Lacerda, Molecular mechanics applied to single-walled carbon nanotubes. Mater Res 2008;11(3):325-33.

13.M. M. Shokrieh, R. Rafiee, Prediction of Young's modulus of graphene sheets and carbon nanotubes using nanoscale continuum mechanics approach. Mater Des 2010;31:790-5.

14.X. $\mathrm{Lu}, \mathrm{Z}$. $\mathrm{Hu}$, Mechanical property evaluation of single-walled carbon nanotubes by finite element modeling. Composites Part B 2012;43:1902-13. 
15.R. Rafiee, M. Heidarhaei, Investigation of chirality and diameter effects on the Young's modulus of carbon nanotubes using non-linear potentials. Compos Struct 2012;94:2460-4.

16.H.-C. Cheng, Y.-L. Liu, Y.-C. Hsu, W.-H. Chen, Atomistic continuum modeling for mechanical properties of single-walled carbon nanotubes. Int J Solids Struct 2009;46:1695-704.

17.M. J. Wernik, S. A. Meguid, Atomistic-based continuum modeling of the nonlinear behavior of carbon nanotubes. Acta Mech 2010;212:167-79. doi: http://dx.doi.org/10.1007/s00707-009-0246-4.

18.G.V. Lier, C.V. Alsenoy, V.V. Doren, P. Geerlings, Ab initio study of the elastic properties of singlewalled carbon nanotubes and grapheme. Chem Phys Lett 2000;326:181-5.

19.K.N. Kudin, G.E. Scuseria, B.I. Yakobson, C2F, BN, and C nanoshell elasticity from ab initio computations. Phys Rev B 2000;64(23):1-10 [Article ID 235406].

20.Y. Y. Zhang, C. M. Wang, Y. Cheng, Y. Xiang, Mechanical properties of bilayer graphene sheets coupled by sp3 bonding, Carbon 2011; 49: 4511-4517.

21.Y. Ren, G. Cao, Effect of geormterical defects on the tensile properties of graphene, Carbon 2016; 103:125-133.

22.S. K. Georgantzinos, G. I. Giannopoulos, N. K. Anifantis, Numerical investigation of elastic mechanical properties of graphene structures, Materials and Design 2010; 31: 4646-4654.

23.S. K. Georgantzinos, D. E. Katsareas, N. K. Anifantis, Graphene characterization: A fully non-linear spring-based finite element prediction, Physica E 2011; 43: 1833-1839..

24.B. Javvaji, P. R. Budarapu , V. K. Sutrakar, D. R. Mahapatra, M. Paggi, G. Zi, T. Rabczuk, Mechanical properties of graphene: Molecular dynamics simulations correlated to continuum based scaling law, Computational Materials Science 2016; 125: 319-327.

25. Chang T, Gao H. Size-dependent elastic properties of a single-walled carbon nanotube via a molecular mechanics model, J of the Mech and Phys of Sol 2003; 51: 1059-1074.

26.W. D. Cornell, P. Cieplak, C. I. Bayly, I. R. Gould, K. M. Merz, D. M. Ferguson, D. C. Spellmeyer, T. Fox, J. W. Caldwell, P. A. Kollman, A second generation force field for the simulation of proteins, nucleic acids, and organic molecules, J of the Amer Chem Soc 1995; 117: 5179-5197

27.T. Belytschko, S. P. Xiao, G. C. Schatz, R. S. Ruoff, Atomistic simulations of nanotube fracture. Phys Rev B 2002; 65(235430):1-8.

28.C. Li, T.-W. Chou, A structural mechanics approach for the analysis of carbon nanotubes, Int J of Sol and Struct 2003; 40: 2487-2499.

29.K. I. Tserpes, P. Papanikos, S. A. Tsirkas, A progressive fracture model for carbon nanotubes, Composites Part B 2006; 37: 662-669.

30.H. Zhao, K. Min, N.R. Aluru, Size and chirality dependent elastic properties of graphene nanoribbons under unixial tension, Nano Letters 2009; 9(8): 3012-3015.

31.M.H. Sadd, Elasticity, Theory, Application and Numerics. Elsevier Butterworth-Heinemann; 2005. 
32.M. Meo, M. Rossi, Prediction of Young's modulus of single wall carbon nanotubes by molecularmechanics based finite element modelling, Composites Science and Technology 2006; 66: 1597-1605.

33.G.I. Giannopoulos, P.A. Kakavas, N.K. Anifantis, Evaluation of the effective mechanical properties of single walled carbon nanotubes using a spring based finite element approach, Computational Materials Science 2008; 41:561-569.

34.B. WengXing, Z. ChangChu, C. WanZhao, Simulation of Young's modulus of single-walled carbon nanotubes by molecular dynamics, Physica B 2004; 352: 156-163.

35.B. T. Kelly, Physics of graphite. London: Applied Science Press; 1981.

36.C. Lee, X. Wei, J. W. Kysar, J. Hone, Measurement of the elastic properties and intrinsic strength of monolayer graphene, Science 2008; 321(5887): 385.

37.B. Peng, M. Locascio, P. Zapol, S. Li, S. L. Mielke, G. C. Schatz, H. D. Espinosa, Measurements of near-ultimate strength for multiwalled carbon nanotubes and irradiation-induced crosslinking improvement, Nature Nanotechnology 2008; 3: 626-631 .

38.F. Hao, D. Fang, Z. Xu, Mechanical and thermal transport properties of graphene with defects. Appl Phys Lett 2011; 99(4):041901-3.

39.J. Zhu, M. He, F. Qiu, Effect of Vacancy Defects on the Young's Modulus and Fracture Strength of Graphene: A Molecular Dynamics Study. Chin. J. Chem. 2012; 30: 1399-1404.

40.A. Ito, S. Okamoto, Molecular dynamics analysis on effects of vacancies upon mechanical properties of graphene and graphite. Eng. Lett. 2012; 20:271-278.

41.A. A. Anastasi, K. Ritos, G. Cassar, M. K. Borg, Mechanical properties of pristine and nanoporous graphene, Molecular Simulation 2016; 42:18, 1502-1511. 